\title{
UNIPOTENT REPRESENTATIONS ATTACHED TO THE PRINCIPAL NILPOTENT ORBIT
}

\author{
LUCAS MASON-BROWN
}

\begin{abstract}
In this paper, we construct and classify the special unipotent representations of a real reductive group attached to the principal nilpotent orbit. We give formulas for the $\mathbf{K}$-types, associated varieties, and Langlands parameters of all such representations.
\end{abstract}

\section{INTRODUCTION}

Let $G$ be the real points of a connected reductive algebraic group. In [1, Adams, Barbasch, and Vogan, following ideas of Arthur ([6], [7]), defined a finite set of irreducible representations of $G$, called special unipotent representations. These representations are conjectured to possess an array of interesting properties (see [1. Chp 1]), including:

(1) They are conjectured to be unitary.

(2) They are conjectured to appear in spaces of automorphic forms.

(3) They are conjectured to generate (through various types of induction) all irreducible unitary representations of $G$ of integral infinitesimal character.

These representations are naturally indexed by special nilpotent orbits for the complexification of $G$. For example, the trivial representation of $G$ is a unipotent representation attached to the nilpotent orbit $\{0\}$. If $G$ is quasi-split, then the spherical principal series representation $\operatorname{Ind}_{B}^{G} \mathbb{C}$ is a unipotent representation attached to the principal nilpotent orbit (there are no other easy examples).

There is no known classification of special unipotent representations. However, properties (1)-(3) above suggest that obtaining one may be an essential ingredient in the classification of the irreducible unitary representations of $G$. In this paper, we will classify the special unipotent representations attached to the principal nilpotent orbit. In fact, we will give two different constructions of such representations:

(1) from (certain) characters of (certain) Borel subgroups using the BeilinsonBernstein construction.

(2) from (approximately) spherical principal series representations of $\theta$-stable parabolic subalgebras using cohomological induction.

The precise statement is given in Corollary 3.6.5. Each construction has its advantages. Construction (1) leads to a simple description of the Langlands parameters of principal unipotent representations (this is done in Section 4). Construction

Received by the editors May 26, 2021.

2020 Mathematics Subject Classification. Primary 22E46.

Key words and phrases. Unipotent representation, nilpotent orbit, Harish-Chandra module, associated variety. 
(2) leads to simple formulas for the associated varieties and $\mathbf{K}$-multiplicities of the representations in question (this is done in Section 3.7).

\section{Preliminaries}

Choose a maximal compact subgroup $K \subset G$ and let $\theta$ be the corresponding Cartan involution of $G$. Denote the (real) Lie algebras of $K$ and $G$ by $\mathfrak{k}_{0}$ and $\mathfrak{g}_{0}$, respectively. There is a Cartan decomposition

$$
\mathfrak{g}_{0}=\mathfrak{k}_{0} \oplus \mathfrak{p}_{0} .
$$

Denote the complexifications of $K$ and $G$ by $\mathbf{K}$ and $\mathbf{G}$, respectively. Denote the complexifications of $\mathfrak{k}_{0}, \mathfrak{g}_{0}$, and $\mathfrak{p}_{0}$ by suppressing the subscripts. Let $\sigma$ be the real form of $\mathbf{G}$ corresponding to $G$. If $\mathfrak{h} \subset \mathfrak{g}$ is a Cartan subalgebra and $\mathfrak{o}$ is an $\mathfrak{h}$ module, write $\Delta(\mathfrak{o}, \mathfrak{h})$ for the multi-set of $\mathfrak{h}$-weights on $\mathfrak{o}$. If $\mathfrak{o}$ is finite-dimensional, let $\rho(\mathfrak{o}):=\frac{1}{2} \sum \Delta(\mathfrak{o}, \mathfrak{h}) \in \mathfrak{h}^{*}$.

2.1. Special unipotent representations. Let $\mathbf{G}^{\vee}$ be the Langlands dual of $\mathbf{G}$. Let $\mathcal{N}_{\mathfrak{g}} \subset \mathfrak{g}$ and $\mathcal{N}_{\mathfrak{g} \vee} \subset \mathfrak{g}^{\vee}$ be the (complex) nilpotent cones. The nilpotent orbits for $\mathbf{G}$ and $\mathbf{G}^{\vee}$ are related by Barbasch-Vogan duality (see [8]). This is a map

$$
d:\left\{\text { nilpotent orbits } \mathcal{O}^{\vee} \subset \mathcal{N}_{\mathfrak{g} \vee}\right\} \rightarrow\left\{\text { nilpotent orbits } \mathcal{O} \subset \mathcal{N}_{\mathfrak{g}}\right\} .
$$

A nilpotent orbit $\mathcal{O} \subset \mathcal{N}_{\mathfrak{g}}$ is special if it lies in the image of $d$.

Every nilpotent $\mathbf{G}^{\vee}$-orbit $\mathcal{O}^{\vee} \subset \mathcal{N}_{\mathfrak{g} \vee}$ gives rise to an infinitesimal character $\lambda_{\mathcal{O}} \vee$ for $U(\mathfrak{g})$ as follows. Let $\mathfrak{h} \subset \mathfrak{g}$ be a Cartan subalgebra and let $\mathfrak{h}^{\vee} \subset \mathfrak{g}^{\vee}$. There is a natural identification $\mathfrak{h}^{\vee} \simeq \mathfrak{h}^{*}$. Choose an element $e^{\vee} \in \mathcal{O}^{\vee}$ and an $\mathfrak{s l}(2)$-triple $\left(e^{\vee}, f^{\vee}, h^{\vee}\right)$. Conjugating by $\mathbf{G}^{\vee}$ if necessary, we can arrange so that $h^{\vee} \in \mathfrak{h}^{\vee} \simeq \mathfrak{h}^{*}$. Put

$$
\lambda_{\mathcal{O}} \vee:=\frac{1}{2} h^{\vee} \in \mathfrak{h}^{\vee} \simeq \mathfrak{h}^{*} .
$$

This element is well-defined modulo the action of the Weyl group and therefore defines an infinitesimal character for $U(\mathfrak{g})$ by means of the Harish-Chandra isomorphism.

Definition 2.1.1. Suppose $\mathcal{O} \subset \mathcal{N}_{\mathfrak{g}}$ is a special nilpotent G-orbit. A unipotent infinitesimal character attached to $\mathcal{O}$ is one of the form $\lambda_{\mathcal{O}^{\vee}}$ for $d\left(\mathcal{O}^{\vee}\right)=\mathcal{O}$. Denote the set of all such $\lambda_{\mathcal{O}} \vee$ by $\operatorname{Unip}_{\mathcal{O}}^{Z}(G)$.

If $I \subset U(\mathfrak{g})$ is a two-sided ideal, let $\operatorname{AV}(I) \subset \mathfrak{g}^{*}$ denote the associated variety of $I$. For notational convenience, we will regard $\mathrm{AV}(I)$ as a subset of $\mathfrak{g}$ (using, for example, any $\mathbf{G}$-invariant identification $\left.\mathfrak{g} \simeq \mathfrak{g}^{*}\right)$. If $I$ is primitive, then $\operatorname{AV}(I)$ is the closure of a single nilpotent orbit $\mathcal{O} \subset \mathcal{N}_{\mathfrak{g}}$ (see [12, Thm 3.10]).

Definition 2.1.2. Suppose $\mathcal{O} \subset \mathcal{N}_{\mathfrak{g}}$ is a special nilpotent G-orbit. A unipotent ideal attached to $\mathcal{O}$ is a primitive ideal $I \subset U(\mathfrak{g})$ such that

(i) The infinitesimal character of $I$ belongs to $\operatorname{Unip}_{\mathcal{O}}^{Z}(G)$.

(ii) $\operatorname{AV}(I)=\overline{\mathcal{O}}$.

Denote the set of such ideals by $\operatorname{Unip}_{\mathcal{O}}^{I}(G)$. A unipotent representation attached to $\mathcal{O}$ is an irreducible $(\mathfrak{g}, \mathbf{K})$-module $X$ such that $\operatorname{Ann}_{U(\mathfrak{g})}(X) \in \operatorname{Unip}_{\mathcal{O}}^{I}(G)$. Denote the set of (isomorphism classes of) such representations by $\operatorname{Unip}_{\mathcal{O}}^{R}(G)$. 
If $\mathcal{O} \subset \mathcal{N}_{\mathfrak{g}}$ is the principal nilpotent orbit, then $d^{-1}(\mathcal{O})$ consists of a single nilpotent $\mathbf{G}^{\vee}$-orbit, $\{0\}$, and so $\operatorname{Unip}_{Z}^{\mathcal{O}}(G)=\{0\}$. Hence, a unipotent ideal attached to $\mathcal{O}$ is a primitive ideal $I \subset U(\mathfrak{g})$ of infinitesimal character 0 and $\operatorname{AV}(I)=\mathcal{N}$. A principal unipotent representation is an irreducible $(\mathfrak{g}, \mathbf{K})$-module which is annihilated by such an ideal.

2.2. Parabolic induction. Let $M(\mathfrak{g}, \mathbf{K})$ be the abelian category of finite-length Harish-Chandra modules and let $K(\mathfrak{g}, \mathbf{K})$ be its Grothendieck group. If $\lambda \in \mathfrak{h}^{*}$, write $M_{\lambda}(\mathfrak{g}, \mathbf{K}) \subset M(\mathfrak{g}, \mathbf{K})$ (resp. $\left.K_{\lambda}(\mathfrak{g}, \mathbf{K}) \subset K(\mathfrak{g}, \mathbf{K})\right)$ for the subcategory (resp. subgroup) of infinitesimal character $\lambda$. Let $\mathcal{N}_{\mathfrak{g}, \theta}=\mathcal{N}_{\mathfrak{g}} \cap \mathfrak{p}$. If $X \in M(\mathfrak{g}, \mathbf{K})$, let $\operatorname{AV}(X) \subset \mathcal{N}_{\mathfrak{g}, \theta}$ denote the associated variety of $X$. By [19, Thm 8.4], there is an equality

$$
\operatorname{AV}(\operatorname{Ann}(X))=\mathbf{G} \operatorname{AV}(X) .
$$

Our notation for parabolic induction is as follows. Let $\mathfrak{q} \subset \mathfrak{g}$ be a parabolic subalgebra with Levi decomposition $\mathfrak{q}=\mathfrak{l} \oplus \mathfrak{u}$ (always, we will assume that $\mathfrak{l}$ is $\theta$-stable). Write $\mathbf{Q}$ and $\mathbf{L}$ for the connected subgroups corresponding to $\mathfrak{q}$ and $\mathfrak{l}$, respectively. Parabolic induction is a left-exact functor (see [13, Chp 2])

$$
\mathbf{I}_{(\mathfrak{l}, \mathbf{L} \cap \mathbf{K})}^{(\mathfrak{g}, \mathbf{K})}: M(\mathfrak{l}, \mathbf{L} \cap \mathbf{K}) \rightarrow M(\mathfrak{g}, \mathbf{K}) .
$$

This functor induces a homomorphism on the corresponding Grothendieck groups $(2.2 .2)$

$$
I(\mathfrak{l}, \mathfrak{q}, \cdot): K(\mathfrak{l}, \mathbf{L} \cap \mathbf{K}) \rightarrow K(\mathfrak{g}, \mathbf{K}) \quad I(\mathfrak{l}, \mathfrak{q},[W]):=\sum_{i}(-1)^{i}\left[R^{i} \mathbf{I}_{(\mathfrak{l}, \mathbf{L} \cap \mathbf{K}) \mathbf{K})}^{(\mathfrak{K})} W\right] .
$$

If $W \in M(\mathfrak{l}, \mathbf{L} \cap \mathbf{K})$ has infinitesimal character $\lambda \in \mathfrak{h}^{*}$, then $R^{i} \mathbf{I}_{(\mathfrak{l}, \mathbf{L} \cap \mathbf{K})}^{(\mathfrak{K}) \mathbf{K})} W$ has infinitesimal character $\lambda+\rho(\mathfrak{u})$, for every $i \geq 0$. Thus, $I(\mathfrak{l}, \mathfrak{q}, \cdot)$ restricts to a homomorphism

$$
I(\mathfrak{l}, \mathfrak{q}, \cdot): K_{\lambda}(\mathfrak{l}, \mathbf{L} \cap \mathbf{K}) \rightarrow K_{\lambda+\rho(\mathfrak{u})}(\mathfrak{g}, \mathbf{K}) .
$$

We will give particular attention to two special cases.

Real parabolic induction: Assume $\mathfrak{q}$ is $\sigma$-stable. Then $Q:=\mathbf{Q}^{\sigma}$ is a real parabolic subgroup. If $W$ is the Harish-Chandra module of a representation $V$ of $L$, then

$$
I_{(\mathfrak{l}, \mathbf{L} \cap \mathbf{K})}^{(\mathfrak{g}, \mathbf{K})} W \simeq \text { Harish-Chandra module of } \operatorname{Ind}_{Q}^{G}(V \otimes|\rho(\mathfrak{u})|),
$$

where $\operatorname{Ind}_{Q}^{G}$ is the usual (analytically-defined) functor of parabolic induction (see [13. Sec 11.2] for a more detailed statement and proof). In particular, $I_{\mathfrak{l}, \mathbf{L} \cap \mathbf{K})}^{(\mathfrak{g}, \mathbf{K})}$ is exact and takes nonzero $(\mathfrak{l}, \mathbf{L} \cap \mathbf{K})$-modules to nonzero $(\mathfrak{g}, \mathbf{K})$-modules.

Cohomological induction: Assume $\mathfrak{q}$ is $\theta$-stable and suppose $W$ is an irreducible

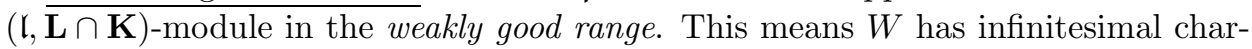
acter $\lambda \in \mathfrak{h}^{*}$ for $\lambda$ satisfying

$$
\operatorname{Re}\left\langle\lambda+\rho(\mathfrak{u}), \alpha^{\vee}\right\rangle \geq 0 \quad \forall \alpha \in \Delta(\mathfrak{u}, \mathfrak{h})
$$

Then there is an integer $t \in \mathbb{Z}_{\geq 0}$ (depending only on $\mathfrak{q}$ ) such that

$$
R^{i} I_{(\mathfrak{l}, \mathbf{L} \cap \mathbf{K})}^{(\mathfrak{g}, \mathbf{K})} W=\left\{\begin{array}{ll}
\text { irreducible or } 0 & i=t \\
0 & i \neq t
\end{array} .\right.
$$

See [13, Thm 0.50] for a proof. If $R^{t} I_{(\mathfrak{l}, \mathbf{L} \cap \mathbf{K})}^{(\mathfrak{g}, \mathbf{K})} W \neq 0$, then

$$
\operatorname{AV}\left(R^{t} I_{(\mathfrak{l}, \mathbf{L} \cap \mathbf{K})}^{(\mathfrak{g}, \mathbf{K})} W\right)=\mathbf{K}(\mathfrak{u} \cap \mathfrak{p}+\operatorname{AV}(W)) \subseteq \mathcal{N}_{\mathfrak{g}, \theta} .
$$


This final fact is well-known to the experts. The proof is a standard argument involving characteristic varieties of $\mathcal{D}$-modules on the flag variety (see e.g. 17, Prop $5.4])$.

\section{Classifichtion of principal unipotent Representations}

\subsection{Beilinson-Bernstein parameters.}

Definition 3.1.1. A Beilinson-Bernstein parameter for $G$ of infinitesimal character 0 is a $\mathbf{K}$-conjugacy class of triples $(\mathfrak{h}, \mathfrak{b}, \chi)$ consisting of

(i) a $\theta$-stable Cartan subalgebra $\mathfrak{h}=\mathfrak{t} \oplus \mathfrak{a} \subset \mathfrak{g}$,

(ii) a Borel subalgebra $\mathfrak{b}=\mathfrak{h} \oplus \mathfrak{n} \subset \mathfrak{g}$, and

(iii) a one-dimensional $(\mathfrak{h}, \mathbf{T})$-module $\chi$ such that $d \chi+\rho(\mathfrak{n})=0$.

Denote the $\mathbf{K}$-conjugacy class of $(\mathfrak{h}, \mathfrak{b}, \chi)$ by $[\mathfrak{h}, \mathfrak{b}, \chi]$ and denote the set of such classes by $\mathrm{BB}_{0}(G)$.

Remark 3.1.2. It is well-known that $\mathrm{BB}_{0}(G)$ is finite; see e.g. [21, Thms 1,2].

Fix a parameter $[\mathfrak{h}, \mathfrak{b}, \chi] \in \mathrm{BB}_{0}(G)$. Replacing $\mathfrak{h}$ by a $\mathbf{K}$-conjugate if necessary, we can (and will) arrange so that $\mathfrak{h}$ is $\sigma$-stable. Thus, $H:=Z_{G}(\mathfrak{h}) \subset G$ is a Cartan subgroup and $(\mathfrak{h}, \mathbf{T})$-modules are in bijection with continuous characters of $H$. Suppose $\alpha \in \Delta(\mathfrak{g}, \mathfrak{h})$ is a real root. Let $\{E, F, D\}$ denote the standard basis of $\mathfrak{s l}_{2}(\mathbb{C})$ (with $D$ semisimple), and fix a Lie algebra embedding $\phi_{\alpha}: \mathfrak{s l}_{2}(\mathbb{R}) \rightarrow \mathfrak{g}_{0}$ such that

$$
\phi_{\alpha}(E) \in \mathfrak{g}_{\alpha} \quad \phi_{\alpha}(F)=-\theta\left(\phi_{\alpha}(E)\right) \quad \phi_{\alpha}(D)=\alpha^{\vee} .
$$

Since $G$ is algebraic, this embedding integrates to a Lie group homomorphism

$$
\Phi_{\alpha}: S L_{2}(\mathbb{R}) \rightarrow G .
$$

Define the element

$$
m_{\alpha}:=\Phi_{\alpha}\left(\begin{array}{cc}
-1 & 0 \\
0 & -1
\end{array}\right) \in T \text {. }
$$

Note that $m_{\alpha}$ is independent of $\phi_{\alpha}$ and $m_{\alpha}^{2}=1$. Thus, $\chi\left(m_{\alpha}\right)= \pm 1$. We say that $\alpha$ is even or odd accordingly. We will impose various special conditions on Beilinson-Bernstein parameters.

Definition 3.1.4. Let $[\mathfrak{h}, \mathfrak{b}, \chi] \in \mathrm{BB}_{0}(G)$. We say that $[\mathfrak{h}, \mathfrak{b}, \chi]$ is

(i) large if every imaginary simple root is noncompact.

(ii) small if every imaginary simple root is compact.

(iii) type $\mathrm{Z}$ if for every complex simple root $\alpha$

$$
\theta(\alpha) \in \Delta^{+}(\mathfrak{g}, \mathfrak{h})
$$

(iv) type $\mathrm{L}$ if for every complex simple root $\alpha$

$$
\theta(\alpha) \in-\Delta^{+}(\mathfrak{g}, \mathfrak{h}) .
$$

(v) totally even if every simple real root $\alpha$ is even for $\chi$.

Given a parameter $[\mathfrak{h}, \mathfrak{b}, \chi] \in \mathrm{BB}_{0}(G)$, there are two naturally defined standard parabolic subalgebras $\mathfrak{q}^{Z}, \mathfrak{q}^{L} \subseteq \mathfrak{g}$. The first, $\mathfrak{q}^{Z}$, is the standard parabolic corresponding to the positive real roots

$$
\mathfrak{l}^{Z}:=\mathfrak{h} \oplus \bigoplus_{\alpha \in \Delta_{\mathbb{R}}} \mathfrak{g}_{\alpha} \quad \mathfrak{u}^{Z}:=\bigoplus_{\alpha \in \Delta^{+} \backslash \Delta_{\mathbb{R}}} \mathfrak{g}_{\alpha} \quad \mathfrak{q}^{Z}:=\mathfrak{l} \oplus \mathfrak{u}
$$


The second, $\mathfrak{q}^{L}$, corresponds to the positive imaginary roots

$$
\mathfrak{l}^{L}:=\mathfrak{h} \oplus \bigoplus_{\alpha \in \Delta_{i \mathbb{R}}} \mathfrak{g}_{\alpha} \quad \mathfrak{u}^{L}:=\bigoplus_{\alpha \in \Delta^{+} \backslash \Delta_{i \mathbb{R}}} \mathfrak{g}_{\alpha} \quad \mathfrak{q}^{L}:=\mathfrak{l} \oplus \mathfrak{u} .
$$

The following is elementary. The proof is left to the reader.

Proposition 3.1.7. In the setting described above

(i) $\mathfrak{q}^{Z}$ is $\theta$-stable if and only if $\Delta^{+}(\mathfrak{g}, \mathfrak{h})$ is type $Z$.

(ii) $\mathfrak{q}^{L}$ is $\sigma$-stable (i.e. real) if and only if $\Delta^{+}(\mathfrak{g}, \mathfrak{h})$ is type $L$.

Definition 3.1.8. A principal unipotent parameter is a Beilinson-Bernstein parameter $[\mathfrak{h}, \mathfrak{b}, \chi] \in \mathrm{BB}_{0}(G)$ which is

(i) large (cf. Definition 3.1.4(i)),

(ii) type $Z$ (cf. Definition 3.1.4(iii)), and

(iii) totally even (cf. Definition 3.1.4(v)).

Denote the set of such parameters by $\operatorname{BB}_{0}^{*}(G)$.

3.2. Cayley transforms. Fix a parameter $[\mathfrak{h}, \mathfrak{b}, \chi] \in \mathrm{BB}_{0}(G)$ and an odd real root $\alpha \in \Delta(\mathfrak{g}, \mathfrak{h})$. Following [20, we will define two new parametrs $c_{\alpha}^{ \pm}[\mathfrak{h}, \mathfrak{b}, \chi] \in \operatorname{BB}_{0}(G)$ called the Cayley transforms of $[\mathfrak{h}, \mathfrak{b}, \chi]$.

Fix an embedding $\phi_{\alpha}: \mathfrak{s l}_{2}(\mathbb{R}) \hookrightarrow \mathfrak{g}_{0}$ of the form described in (3.1.3). Consider the 'compact' basis of $\mathfrak{s l}_{2}(\mathbb{C})$

$$
E_{c}=\frac{1}{2}\left(\begin{array}{cc}
1 & -i \\
-i & -1
\end{array}\right) \quad F_{c}=\frac{1}{2}\left(\begin{array}{cc}
1 & i \\
i & -1
\end{array}\right) \quad D_{c}=\left(\begin{array}{cc}
0 & i \\
-i & 0
\end{array}\right)
$$

Define a $\theta$-stable Cartan subalgebra $\mathfrak{h}_{0}^{\alpha}$ of $\mathfrak{g}_{0}$

$$
\mathfrak{t}_{0}^{\alpha}:=\mathfrak{t}_{0} \oplus i \mathbb{R} \phi_{\alpha}\left(D_{c}\right) \quad \mathfrak{a}_{0}^{\alpha}:=\operatorname{ker} \alpha \cap \mathfrak{a}_{0} \quad \mathfrak{h}_{0}^{\alpha}:=\mathfrak{t}_{0}^{\alpha} \oplus \mathfrak{a}_{0}^{\alpha} .
$$

Let $H^{\alpha}$ be the corresponding Cartan subgroup of $G$

$$
T^{\alpha}:=Z_{K}\left(\mathfrak{t}_{0}^{\alpha}\right) \quad A:=\exp \left(\mathfrak{a}_{0}^{\alpha}\right) \quad H^{\alpha}:=T^{\alpha} A^{\alpha} .
$$

Write $c_{\alpha}^{ \pm}: \mathfrak{g} \rightarrow \mathfrak{g}$ for the Cayley transforms corresponding to $\alpha$

$$
c_{\alpha}^{ \pm}:=\exp \left(\operatorname{ad}\left(\frac{ \pm i \pi}{4} \phi_{\alpha}(E+F)\right)\right) .
$$

By construction, $c_{\alpha}^{ \pm} \mathfrak{h}=\mathfrak{h}^{\alpha}$.

Define two characters $\tau_{ \pm 1}$ of $S O_{2}(\mathbb{R})$ by

$$
d \tau_{ \pm 1}\left(\begin{array}{cc}
0 & 1 \\
-1 & 0
\end{array}\right)= \pm i
$$

Since $\phi_{\alpha}$ is injective $\operatorname{ker} \Phi_{\alpha} \subseteq\{ \pm 1\}$. Since $\alpha$ is odd, $\Phi_{\alpha}(-1)=m_{\alpha} \neq 1$ and hence $\Phi_{\alpha}$ is injective. Define characters $c_{\alpha}^{ \pm} \chi$ of the product group $\Phi_{\alpha}\left(S O_{2}(\mathbb{R})\right) \times T_{1}^{\alpha}$ by the formulas

$$
c_{\alpha}^{ \pm} \chi\left(\Phi_{\alpha}(g), t\right)=\tau_{ \pm 1}(g) \chi(t)
$$

The multiplication map

$$
\Phi_{\alpha}\left(S O_{2}(\mathbb{R})\right) \times T_{1}^{\alpha} \rightarrow T^{\alpha}
$$

is surjective with kernel $\left\{(1,1),\left(m_{\alpha}, m_{\alpha}\right)\right\}$ (see [18, Lem 8.3.13]). Since $\alpha$ is odd,

$$
c_{\alpha}^{ \pm} \chi\left(m_{\alpha}, m_{\alpha}\right)=(-1)^{2}=1,
$$


and therefore both characters $c_{\alpha}^{ \pm} \chi$ descend to characters of $T^{\alpha}$. We can regard these characters as one-dimensional $\left(\mathfrak{h}^{\alpha}, \mathbf{T}^{\alpha}\right)$-modules by

$$
d c_{\alpha}^{ \pm}(t+a)=d c_{\alpha}^{ \pm}(t)+\chi(a) \quad t \in \mathfrak{t}^{\alpha}, a \in \mathfrak{a}^{\alpha} \subset \mathfrak{a} .
$$

The Cayley transforms of $[\mathfrak{h}, \mathfrak{b}, \chi]$ are the parameters

$$
c_{\alpha}^{ \pm}[\mathfrak{h}, \mathfrak{b}, \chi]:=\left[\mathfrak{h}^{\alpha}, c_{\alpha}^{ \pm} \mathfrak{b}, \chi_{\alpha}^{ \pm} \chi\right] \in \mathrm{BB}_{0}(G) .
$$

We will have reason to consider the following function on $\mathrm{BB}_{0}(G)$

$$
d: \mathrm{BB}_{0}(G) \rightarrow \mathbb{Z}_{\geq 0} \quad d[\mathfrak{h}, \mathfrak{b}, \chi]:=\operatorname{dim}(\mathfrak{b} \cap \mathfrak{k}) .
$$

The following is elementary. Its proof is left to the reader.

Lemma 3.2.3. Let $[\mathfrak{h}, \mathfrak{b}, \chi] \in \mathrm{BB}_{0}(G)$ and let $\alpha$ be an odd simple real root. Then

$$
d\left(c_{\alpha}^{ \pm}[\mathfrak{h}, \mathfrak{b}, \chi]\right)=d[\mathfrak{h}, \mathfrak{b}, \chi]+1 .
$$

3.3. Cross actions. Fix a parameter $[\mathfrak{h}, \mathfrak{b}, \chi] \in \mathrm{BB}_{0}(G)$ and a simple root $\alpha \in$ $\Delta^{+}(\mathfrak{g}, \mathfrak{h})$. Following [20], we will define a new parameter $s_{\alpha}[\mathfrak{h}, \mathfrak{b}, \chi]$ called the cross action of $[\mathfrak{h}, \mathfrak{b}, \chi]$. Let $s_{\alpha} \in W$ be the simple reflection corresponding to $\alpha$. Let $s_{\alpha} \mathfrak{b} \subset \mathfrak{g}$ be the Borel subalgebra corresponding to the positive system

$$
s_{\alpha} \Delta^{+}(\mathfrak{g}, \mathfrak{h})=\Delta^{+}(\mathfrak{g}, \mathfrak{h}) \cup\{-\alpha\} \backslash\{\alpha\} .
$$

The cross action of $\alpha$ on $[\mathfrak{h}, \mathfrak{b}, \chi \otimes \alpha]$ is the parameter

$$
s_{\alpha}[\mathfrak{h}, \mathfrak{b}, \chi]:=\left[\mathfrak{h}, s_{\alpha} \mathfrak{b}, \chi \otimes \alpha\right] \in \mathrm{BB}_{0}(G) .
$$

Analogous to Lemma 3.2.3, we have the following elementary result. Its proof is left to the reader.

Lemma 3.3.1. Let $[\mathfrak{h}, \mathfrak{b}, \chi] \in \mathrm{BB}_{0}(G)$ and let $\alpha \in \Delta^{+}(\mathfrak{g}, \mathfrak{h})$ be a complex simple root.

(i) If $\theta(\alpha) \in-\Delta^{+}(\mathfrak{g}, \mathfrak{h})$, then

$$
d\left(s_{\alpha}[\mathfrak{h}, \mathfrak{b}, \chi]\right)=d[\mathfrak{h}, \mathfrak{b}, \chi]+1 .
$$

(ii) If $\theta(\alpha) \in \Delta^{+}(\mathfrak{g}, \mathfrak{h})$, then

$$
d\left(s_{\alpha}[\mathfrak{h}, \mathfrak{b}, \chi]\right)=d[\mathfrak{h}, \mathfrak{b}, \chi]-1 .
$$

3.4. Parabolic induction of Beilinson-Bernstein parameters. If $(\mathfrak{h}, \mathfrak{b}, \chi)$ is a triple as in Definition 3.1.1, then $I(\mathfrak{h}, \mathfrak{b}, \chi)$ is a virtual finite-length $(\mathfrak{g}, \mathbf{K})$-module of infinitesimal character 0 . The assignment $(\mathfrak{h}, \mathfrak{b}, \chi) \mapsto I(\mathfrak{h}, \mathfrak{b}, \chi)$ is constant on $\mathbf{K}$-conjugacy classes and thus induces a function

$$
I: \mathrm{BB}_{0}(G) \rightarrow K_{0}(\mathfrak{g}, \mathbf{K}) .
$$

This function can be described in a different way using the Beilinson-Bernstein construction. Below, we will summarize the main ideas and consequences (for more details and proofs, we refer the reader to [10] or [11, Chp 11]).

Let $\mathcal{B}=\{\mathfrak{b} \subset \mathfrak{g}\}$ be the flag variety for $\mathbf{G}$. Consider the the sheaf of twisted differential operators $\mathcal{D}_{-\rho}$ on $\mathcal{B}$ corresponding to the functional $-\rho \in \mathfrak{h}^{*}$, and consider the abelian category $M\left(\mathcal{D}_{-\rho}, \mathbf{K}\right)$ of $\mathbf{K}$-equivariant quasi-coherent $\mathcal{D}_{-} \rho_{\text {-modules on }}$ $\mathcal{B}$. The irreducible objects in this category are parameterized by $\mathrm{BB}_{0}(G)$. The correspondence is as follows. Fix a parameter $[\mathfrak{h}, \mathfrak{b}, \chi] \in \mathrm{BB}_{0}(G)$. The Borel subalgebra $\mathfrak{b}$ determines a $\mathbf{K}$-orbit $Z=\mathbf{K} \cdot \mathfrak{b} \subset \mathcal{B}$. Denote the locally-closed embedding by $j: Z \subset \mathcal{B}$. On the $\mathbf{K}$-orbit $Z$, there is a sheaf of TDOs $\mathcal{D}_{-\rho}^{Z}$ obtained by restricting $\mathcal{D}_{-\rho}$ along $Z \subset \mathcal{B}$, and the one-dimensional (h, T)-module 
$\chi$ determines an irreducible object $\mathcal{L}_{\chi} \in M\left(\mathcal{D}_{-\rho}^{Z}, \mathbf{K}\right)$. There is a left-exact functor $j_{!}: M\left(\mathcal{D}_{-\rho}^{Z}, \mathbf{K}\right) \rightarrow M\left(\mathcal{D}_{-\rho}, \mathbf{K}\right)$ called the exceptional pushforward. The object $j_{!} \mathcal{L}_{\chi} \in M\left(\mathcal{D}_{-\rho}, \mathbf{K}\right)$ contains a unique irreducible subobject. This defines a bijection

$$
\begin{aligned}
\mathrm{BB}_{0}(G) & \left.\stackrel{\sim}{\rightarrow} \text { irreducibles in } M\left(\mathcal{D}_{-\rho}, \mathbf{K}\right)\right\} \\
{[\mathfrak{h}, \mathfrak{b}, \chi] } & \mapsto \text { unique irreducible subobject in } j_{!} \mathcal{L}_{\chi} .
\end{aligned}
$$

The $\mathbf{G}$-action on $\mathcal{B}$ induces an algebra homomorphism

$$
\phi: U(\mathfrak{g}) \rightarrow \Gamma\left(\mathcal{B}, \mathcal{D}_{-\rho}\right) .
$$

This map is surjective with kernel equal to the two-sided ideal generated by the kernel of the infinitesimal character corresponding to 0 . If $\mathcal{M} \in M\left(\mathcal{D}_{-\rho}, \mathbf{K}\right)$, then $\Gamma(\mathcal{B}, \mathcal{M})$ can be regarded (using $\phi$ ) as a finite-length $(\mathfrak{g}, \mathbf{K})$-module of infinitesimal character 0 . This defines a functor

$$
\Gamma: M\left(\mathcal{D}_{-\rho}, \mathbf{K}\right) \rightarrow M_{0}(\mathfrak{g}, \mathbf{K}) .
$$

Since $0 \in \mathfrak{h}^{*}$ is integrally dominant, $\Gamma$ is reasonably well-behaved. The following result is well-known. A proof can be found in [11, Cor 11.2.6].

Theorem 3.4.1. $\Gamma$ is an exact functor, and there is a bijection

$\Gamma:\left\{\right.$ irreducibles $\mathcal{M} \in M\left(\mathcal{D}_{-\rho}, \mathbf{K}\right)$ with $\left.\Gamma(M) \neq 0\right\} \stackrel{\sim}{\longrightarrow}\left\{\right.$ irreducibles $\left.M \in M_{0}(\mathfrak{g}, \mathbf{K})\right\}$.

The classes $I[\mathfrak{h}, \mathfrak{b}, \chi]$ and $\Gamma\left(\mathcal{B}, j_{!} \mathcal{L}_{\chi}\right)$ are known to (essentially) coincide. The following is a special case of the Duality Theorem of Hecht, Milicic, Schmid, and Wolf (see [10, Thm 4.3]).

Theorem 3.4.2. Suppose $[\mathfrak{h}, \mathfrak{b}, \chi] \in \mathrm{BB}_{0}(G)$. Then

$$
\left[\Gamma\left(\mathcal{B}, j_{!} \mathcal{L}_{\chi}\right)\right]= \pm I[\mathfrak{h}, \mathfrak{b}, \chi] \in K_{0}(\mathfrak{g}, \mathbf{K}) .
$$

Corollary 3.4.3. Let $\left[\mathfrak{h}_{1}, \mathfrak{b}_{1}, \chi_{1}\right],\left[\mathfrak{h}_{2}, \mathfrak{b}_{2}, \chi_{2}\right] \in \mathrm{BB}_{0}(G)$. Suppose $I\left[\mathfrak{h}_{1}, \mathfrak{b}_{1}, \chi_{1}\right]$ and $I\left[\mathfrak{h}_{2}, \mathfrak{b}_{2}, \chi_{2}\right]$ are nonzero and irreducible, and that

$$
I\left[\mathfrak{h}_{1}, \mathfrak{b}_{1}, \chi_{1}\right]= \pm I\left[\mathfrak{h}_{2}, \mathfrak{b}_{2}, \chi_{2}\right] .
$$

Then $\left[\mathfrak{h}_{1}, \mathfrak{b}_{1}, \chi_{1}\right]=\left[\mathfrak{h}_{2}, \mathfrak{b}_{2}, \chi_{2}\right]$.

We conclude this section by examining the behavior of the function $I: \mathrm{BB}_{0}(G) \rightarrow$ $K_{0}(\mathfrak{g}, \mathbf{K})$ under Cayley transforms and cross actions. The following result is an immediate consequence of the Transfer Theorem of Knapp and Vogan (see [13, Thm 11.87]).

Theorem 3.4.4. Suppose $\alpha \in \Delta^{+}(\mathfrak{g}, \mathfrak{h})$ is a complex simple root. Then

$$
I[\mathfrak{h}, \mathfrak{b}, \chi]=-I\left(s_{\alpha}[\mathfrak{h}, \mathfrak{b}, \chi]\right) .
$$

For Cayley transforms, the situation is more subtle. The image of $T$ under $\alpha$ is a compact subgroup of $\mathbb{R}^{\times}$, and hence a subgroup of $\{ \pm 1\}$.

Definition 3.4.5 ([18], Def 8.3.4). We say that $\alpha$ is type 1 (resp. type 2) if $\alpha(T)=\{1\}(\operatorname{resp} . \alpha(T)=\{ \pm 1\})$.

We will prove the following result. 
Theorem 3.4.6. Let $[\mathfrak{h}, \mathfrak{b}, \chi] \in \mathrm{BB}_{0}(G)$ and let $\alpha \in \Delta^{+}(\mathfrak{g}, H)$ be an odd simple real root. Then

(i) If $\alpha$ is type 1 ,

$$
I[\mathfrak{h}, \mathfrak{b}, \chi]=-I\left(c_{\alpha}^{+}[\mathfrak{h}, \mathfrak{b}, \chi]\right)-I\left(c_{\alpha}^{-}[\mathfrak{h}, \mathfrak{b}, \chi]\right) .
$$

(ii) If $\alpha$ is type 2 ,

$$
I[\mathfrak{h}, \mathfrak{b}, \chi]=-I\left(c_{\alpha}^{+}[\mathfrak{h}, \mathfrak{b}, \chi]\right)=-I\left(c_{\alpha}^{-}[\mathfrak{h}, \mathfrak{b}, \chi]\right) .
$$

To simplify the notation, let

$$
G^{s}:=S L_{2}(\mathbb{R}) \quad K^{s}:=S O_{2}(\mathbb{R}) \quad H^{s}:=\left\{\left(\begin{array}{cc}
t & 0 \\
0 & t^{-1}
\end{array}\right)\right\} \quad T^{s}:=\{ \pm \mathrm{Id}\} .
$$

Let $\tau_{ \pm 1}$ be the characters of $K^{s}$ defined in (3.2.1). Let $\mathfrak{b}^{s} \subset \mathfrak{g}^{s}$ be the Borel subalgebra of upper triangular matrices, and let $\mathfrak{b}_{c}^{s}( \pm) \subset \mathfrak{g}^{s}$ be the Borel subalgebras containing $\mathfrak{k}^{s}$. Arrange the signs so that

$$
d \tau_{1}=-\rho\left(\mathfrak{n}_{c}^{s}(+)\right) \quad d \tau_{-1}=-\rho\left(\mathfrak{n}_{c}^{s}(-)\right) .
$$

Consider the Levi subgroup $L_{\alpha}:=Z_{G}(\operatorname{ker} \alpha)$, and the Borel subalgebras

$$
\mathfrak{b}^{\mathfrak{l}_{\alpha}}:=\mathfrak{b} \cap \mathfrak{l}_{\alpha} \supset \mathfrak{h} \quad \mathfrak{b}_{c}^{\mathfrak{l}_{\alpha}}( \pm)=c_{\alpha}^{ \pm} \mathfrak{b} \cap \mathfrak{l}_{\alpha} \supset c_{\alpha} \mathfrak{h} .
$$

Since $\Phi_{\alpha}\left(G^{s}\right)$ centralizes ker $\alpha$, there is an isogeny

$$
r_{\alpha}: G^{s} \times \operatorname{ker} \alpha \rightarrow L_{\alpha} \quad r_{\alpha}(g, h)=\Phi_{\alpha}(g) h
$$

with kernel $\left\{(1,1),\left(-1, m_{\alpha}\right)\right\}$. Consider the subgroups

$$
\begin{array}{ll}
\bar{L}_{\alpha}:=r_{\alpha}\left(G^{s} \times \operatorname{ker} \alpha\right) \subseteq L_{\alpha} & \bar{H}:=r_{\alpha}\left(H^{s} \times \operatorname{ker} \alpha\right) \subseteq H \\
\bar{T}:=r_{\alpha}\left(T^{s} \times \operatorname{ker} \alpha\right) \subseteq T & \bar{H}^{\alpha}:=r_{\alpha}\left(K^{s} \times \operatorname{ker} \alpha\right) \subset H^{\alpha} .
\end{array}
$$

If $\alpha$ is type 1 , then these inclusions are equalities. If $\alpha$ is type 2 , then they are index 2 subgroups (see [18, Lem 8.3.13]). As an easy consequence, we obtain the following structural fact.

Lemma 3.4.7. If $\alpha$ is type 1 , then $\mathfrak{b}_{c}^{\mathfrak{l}_{\alpha}}( \pm)$ are non-conjugate under $L_{\alpha}$. If $\alpha$ is type 2, then for any element $t \in T$ with $\alpha(t)=-1, \operatorname{Ad}(t)$ acts by inversion on $\Phi_{\alpha}\left(K^{s}\right)$ and interchanges $\mathfrak{b}_{c}^{\mathfrak{l}_{\alpha}}( \pm)$.

Proposition 3.4.8. Let $[\mathfrak{h}, \mathfrak{b}, \chi] \in \mathrm{BB}_{0}(G)$ and let $\alpha \in \Delta^{+}(\mathfrak{g}, H)$ be an odd real simple root. Then

(i) If $\alpha$ is type 1 , there is an equality in $K\left(\mathfrak{l}_{\alpha}, \mathbf{L}_{\alpha} \cap \mathbf{K}\right)$

$$
I\left[\mathfrak{h}, \mathfrak{b}^{\mathfrak{l}_{\alpha}}, \chi\right]=-I\left[\mathfrak{h}^{\alpha}, \mathfrak{b}_{c}^{\mathfrak{l}_{\alpha}}(+), c_{\alpha}^{+} \chi\right]-I\left[\mathfrak{h}^{\alpha}, \mathfrak{b}_{c}^{\mathfrak{l}_{\alpha}}(-), c_{\alpha}^{-} \chi\right],
$$

and the terms on the right are irreducible.

(ii) If $\alpha$ is type 2 , there are equalities in $K\left(\mathfrak{l}_{\alpha}, \mathbf{L}_{\alpha} \cap \mathbf{K}\right)$

$$
I\left[\mathfrak{h}, \mathfrak{b}^{\mathfrak{l}_{\alpha}}, \chi\right]=-I\left[\mathfrak{h}^{\alpha}, \mathfrak{b}_{c}^{\mathfrak{l}_{\alpha}}(+), c_{\alpha}^{+} \chi\right]=-I\left[\mathfrak{h}^{\alpha}, \mathfrak{b}_{c}^{\mathfrak{l}_{\alpha}}(-), c_{\alpha}^{-} \chi\right],
$$

and all terms are irreducible.

Proof. First, assume $\alpha$ is type 1. Consider the pullback $r_{\alpha}^{*} \chi$ of $\chi$ along $r_{\alpha}: H^{s} \times$ $\operatorname{ker} \alpha \rightarrow H$. This character has the form

$$
r_{\alpha}^{*} \chi=\left.\tau \otimes \chi\right|_{\operatorname{ker} \alpha}
$$

for some character $\tau$ of $H^{s}$. Since $\alpha$ is odd, $\tau(-1)=-1$, and since $d \chi=-\rho(\mathfrak{n})$,

$$
d \tau(D)=d \chi\left(\alpha^{\vee}\right)=-\rho(\mathfrak{n})\left(\alpha^{\vee}\right)=-1 .
$$


Hence, $\tau$ is the character

$$
\tau: H^{s} \rightarrow \mathbb{R}^{\times} \quad \tau\left(\left(\begin{array}{cc}
t & 0 \\
0 & t^{-1}
\end{array}\right)=t^{-1} .\right.
$$

For $G^{s}$, there is a well-known decomposition (of the non-spherical principal series of infinitesimal character 0 into two limit of discrete series)

$$
I\left[\mathfrak{h}^{s}, \mathfrak{b}^{s}, \tau\right]=-I\left[\mathfrak{k}^{s}, \mathfrak{b}_{c}^{s}(+), \tau_{1}\right]-I\left[\mathfrak{k}^{s}, \mathfrak{b}_{c}^{s}(-), \tau_{-1}\right] .
$$

Hence $I\left[\mathfrak{h}^{s} \times \operatorname{ker} \alpha, \mathfrak{b}^{s} \oplus \operatorname{ker} \alpha, r_{\alpha}^{*} \chi\right]$ decomposes into irreducible classes

$$
\begin{aligned}
I\left[\mathfrak{h}^{s}\right. & \left.\times \operatorname{ker} \alpha, \mathfrak{b}^{s} \oplus \operatorname{ker} \alpha, r_{\alpha}^{*} \chi\right] \\
& =\left.I\left[\mathfrak{h}^{s}, \mathfrak{b}^{s}, \tau\right] \otimes \chi\right|_{\operatorname{ker} \alpha} \\
& =-\left.\left(I\left[\mathfrak{k}^{s}, \mathfrak{b}_{c}^{s}(+), \tau_{1}\right]+I\left[\mathfrak{k}^{s}, \mathfrak{b}_{c}^{s}(-), \tau_{-1}\right]\right) \otimes \chi\right|_{\operatorname{ker} \alpha} \\
& =-I\left[\mathfrak{k}^{s} \times \operatorname{ker} \alpha, \mathfrak{b}_{c}^{s}(+) \oplus \operatorname{ker} \alpha, \tau_{1} \otimes \chi\right]-I\left[\mathfrak{k}^{s} \times \operatorname{ker} \alpha, \mathfrak{b}_{c}^{s}(-) \oplus \operatorname{ker} \alpha, \tau_{-1} \otimes \chi\right] \\
& =-I\left[\mathfrak{k}^{s} \times \operatorname{ker} \alpha, \mathfrak{b}_{c}^{s}(+) \oplus \operatorname{ker} \alpha, r_{\alpha}^{*} c_{\alpha}^{+} \chi\right]-I\left[\mathfrak{k}^{s} \times \operatorname{ker} \alpha, \mathfrak{b}_{c}^{s}(-) \oplus \operatorname{ker} \alpha, r_{\alpha}^{*} c_{\alpha}^{-} \chi\right] .
\end{aligned}
$$

Since $\alpha$ is type $1, r_{\alpha}$ is surjective. Hence, $I$ and $r_{\alpha}^{*}$ commute. Moving $r_{\alpha}^{*}$ past $I$ in the equation above, we get

$$
r_{\alpha}^{*} I\left[\mathfrak{h}, \mathfrak{b}^{\mathfrak{l}_{\alpha}}, \chi\right]=-r_{\alpha}^{*} I\left[\mathfrak{h}^{\alpha}, \mathfrak{b}_{c}^{\mathfrak{l}_{\alpha}}(+), c_{\alpha}^{+} \chi\right]-r_{\alpha}^{*} I\left[\mathfrak{h}^{\alpha}, \mathfrak{b}_{c}^{\mathfrak{l}_{\alpha}}(-), c_{\alpha}^{-} \chi\right],
$$

which implies

$$
I\left[\mathfrak{h}, \mathfrak{b}^{\mathfrak{l}_{\alpha}}, \chi\right]=-I\left[\mathfrak{h}^{\alpha}, \mathfrak{b}_{c}^{\mathfrak{l}_{\alpha}}(+), c_{\alpha}^{+} \chi\right]-I\left[\mathfrak{h}^{\alpha}, \mathfrak{b}_{c}^{\mathfrak{l}_{\alpha}}(-), c_{\alpha}^{-} \chi\right],
$$

proving part (i) of the proposition.

Now suppose $\alpha$ is type 2. By the argument above, there is a decomposition in $K\left(\mathfrak{l}_{\alpha}, \bar{L}_{\alpha} \cap K\right)$

$$
I\left[\overline{\mathfrak{h}}, \mathfrak{b}^{\mathfrak{l}_{\alpha}}, \chi\right]=-I\left[\mathfrak{h}^{\alpha}, \mathfrak{b}_{c}^{\mathfrak{l}_{\alpha}}(+), c_{\alpha}^{+} \chi\right]-I\left[\mathfrak{h}^{\alpha}, \mathfrak{b}_{c}^{\mathfrak{l}_{\alpha}}(-), c_{\alpha}^{-} \chi\right] .
$$

Let $\epsilon$ be the (unique) nontrivial one-dimensional $(\mathfrak{h}, T)$-module with trivial restriction to $(\mathfrak{h}, \bar{T})$. Then $I_{(\mathfrak{h}, \bar{T})}^{(\mathfrak{h}, T)} \chi \simeq \chi \oplus(\chi \otimes \epsilon)$ and (3.4.9) becomes

$$
I\left[\mathfrak{h}, \mathfrak{b}^{\mathfrak{l}_{\alpha}}, \chi\right]+I\left[\mathfrak{h}, \mathfrak{b}^{\mathfrak{l}_{\alpha}}, \chi \otimes \epsilon\right]=-I\left[\mathfrak{h}^{\alpha}, \mathfrak{b}_{c}^{\mathfrak{l}_{\alpha}}(+), c_{\alpha}^{+} \chi\right]-I\left[\mathfrak{h}^{\alpha}, \mathfrak{b}_{c}^{\mathfrak{l}_{\alpha}}(-), c_{\alpha}^{-} \chi\right] .
$$

By Lemma 3.4.7, the two-element quotient group $\left(L_{\alpha} \cap K\right) /\left(\bar{L}_{\alpha} \cap K\right)$ exchanges the terms on the right. Hence by Clifford theory, they are isomorphic. If we apply the (exact) functor $I_{\left(\mathfrak{r}_{\alpha}, \bar{L}_{\alpha} \cap K\right)}^{\left(\mathfrak{l}_{\alpha}, L_{\alpha} \cap K\right)}$ to both sides of (3.4.10), we obtain a decomposition in $K\left(\mathfrak{l}_{\alpha}, L_{\alpha} \cap K\right)$

$$
I\left[\mathfrak{h}, \mathfrak{b}^{\mathfrak{l}_{\alpha}}, \chi\right]+I\left[\mathfrak{h}, \mathfrak{b}^{\mathfrak{l}_{\alpha}}, \chi \otimes \epsilon\right]=-I\left[\mathfrak{h}^{\alpha}, \mathfrak{b}_{c}^{\mathfrak{l}_{\alpha}}(+), c_{\alpha}^{+} \chi\right]-I\left[\mathfrak{h}^{\alpha}, \mathfrak{b}_{c}^{\mathfrak{l}_{\alpha}}(-), c_{\alpha}^{-} \chi\right] .
$$

Since all terms are irreducible, this implies

$$
I\left[\mathfrak{h}, \mathfrak{b}^{\mathfrak{l}_{\alpha}}, \chi\right]=I\left[\mathfrak{h}, \mathfrak{b}^{\mathfrak{l}_{\alpha}}, \chi \otimes \epsilon\right]=-I\left[\mathfrak{h}^{\alpha}, \mathfrak{b}_{c}^{\mathfrak{l}_{\alpha}}(+), c_{\alpha}^{+} \chi\right]=-I\left[\mathfrak{h}^{\alpha}, \mathfrak{b}_{c}^{\mathfrak{l}_{\alpha}}(-), c_{\alpha}^{-} \chi\right],
$$

which proves part (ii) of the proposition.

We are now prepared to prove Theorem 3.4.6.

Proof of Theorem 3.4.6. Recall the parabolic subalgebra $\mathfrak{q}^{Z}=\mathfrak{l}^{Z} \oplus \mathfrak{u}^{Z}$ defined in (3.1.5). Note that $\alpha$ is an odd simple root for the positive system $\Delta^{+}\left(\mathfrak{l}^{Z}, \mathfrak{h}\right)$. Let $\mathfrak{p}_{\alpha}=\mathfrak{l}_{\alpha} \oplus \mathfrak{u}_{\alpha} \subset \mathfrak{l}$ be the corresponding minimal parabolic. By definition

$$
\mathfrak{b}=\mathfrak{b}^{\mathfrak{l}_{\alpha}} \oplus \mathfrak{u}_{\alpha} \oplus \mathfrak{u}^{Z} \quad c_{\alpha}^{ \pm} \mathfrak{b}=\mathfrak{b}_{c}^{\mathfrak{l}_{\alpha}}( \pm) \oplus \mathfrak{u}_{\alpha} \oplus \mathfrak{u}^{Z} .
$$


Suppose $\alpha$ is type 1. By Proposition 3.4.8 and the transitivity of induction

$$
\begin{aligned}
& I[\mathfrak{h}, \mathfrak{b}, \chi] \\
& \left.\quad=I\left[\mathfrak{l}^{Z}, \mathfrak{q}^{Z}, I\left[L_{\alpha}, \mathfrak{p}_{\alpha}, I\left[\mathfrak{h}, \mathfrak{b}^{\mathfrak{l}_{\alpha}}, \chi\right]\right]\right]\right) \\
& \quad=-I\left[\mathfrak{l}^{Z}, \mathfrak{q}^{Z}, I\left[\mathfrak{l}_{\alpha}, \mathfrak{p}_{\alpha}, I\left[\mathfrak{h}^{\alpha}, \mathfrak{b}_{c}^{\mathfrak{l}_{\alpha}}(+), c_{\alpha}^{+} \chi\right]\right]\right]-I\left[\mathfrak{l}^{Z}, \mathfrak{q}^{Z}, I\left[\mathfrak{l}_{\alpha}, \mathfrak{p}_{\alpha}, I\left[\mathfrak{h}^{\alpha}, \mathfrak{b}_{c}^{\mathfrak{l}_{\alpha}}(-), c_{\alpha}^{-} \chi\right]\right]\right] \\
& \quad=-I\left[\mathfrak{h}^{\alpha}, c_{\alpha}^{+} \mathfrak{b}, c_{\alpha}^{+} \chi\right]-I\left[\mathfrak{h}^{\alpha}, c_{\alpha}^{-} \mathfrak{b}, c_{\alpha}^{-} \chi\right] \\
& \quad=-I\left(c_{\alpha}^{+}[\mathfrak{h}, \mathfrak{b}, \chi]\right)-I\left(c_{\alpha}^{-}[\mathfrak{h}, \mathfrak{b}, \chi]\right) .
\end{aligned}
$$

If $\alpha$ is type 2 , we obtain

$$
\begin{aligned}
I[\mathfrak{h}, \mathfrak{b}, \chi] & =I\left[\mathfrak{l}^{Z}, \mathfrak{q}^{Z}, I\left[\mathfrak{l}_{\alpha}, \mathfrak{p}_{\alpha}, I\left[\mathfrak{h}, \mathfrak{b}^{\mathfrak{l}_{\alpha}}, \chi\right]\right]\right] \\
& =-I\left[\mathfrak{l}^{Z}, \mathfrak{q}^{Z}, I\left[\mathfrak{l}_{\alpha}, \mathfrak{p}_{\alpha}, I\left[\mathfrak{h}^{\alpha}, \mathfrak{b}_{c}^{\mathfrak{l}_{\alpha}}( \pm), c_{\alpha}^{ \pm} \chi\right]\right]\right. \\
& =-I\left[\mathfrak{h}^{\alpha}, c_{\alpha}^{ \pm} \mathfrak{b}, c_{\alpha}^{ \pm} \chi\right] \\
& =-I\left(c_{\alpha}^{ \pm}[\mathfrak{h}, \mathfrak{b}, \chi]\right) .
\end{aligned}
$$

3.5. Zuckerman parameters. In this section, we define our second set of parameters for principal unipotent representations.

Definition 3.5.1. A Zuckerman parameter of infinitesimal character 0 is a $\mathbf{K}$ conjugacy class of triples $\left(\mathfrak{l}, \mathfrak{q}, \chi^{\#}\right)$ consisting of

(i) a $\theta$-stable Levi subalgebra $\mathfrak{l} \subset \mathfrak{g}$, split modulo center,

(ii) a $\theta$-stable parabolic subalgebra $\mathfrak{q} \subset \mathfrak{g}$ containing $\mathfrak{l}$ as a Levi subalgebra, and

(iii) a one-dimensional $(\mathfrak{l}, \mathbf{L} \cap \mathbf{K})$-module $\chi^{\#}$ satisfying $d \chi^{\#}=-\rho(\mathfrak{u})$.

Denote the $\mathbf{K}$-conjugacy class of $\left(\mathfrak{l}, \mathfrak{q}, \chi^{\#}\right)$ by $\left[\mathfrak{l}, \mathfrak{q}, \chi^{\#}\right]$ and denote the set of all such conjugacy classes by $\mathrm{Z}_{0}(G)$. A Zuckerman parameter is unipotent if it satisfies the additional condition:

(iv) $\mathfrak{u} \cap \mathfrak{p}+\mathcal{N}_{\mathfrak{l}, \theta}$ contains a principal nilpotent element of $\mathfrak{g}$.

Write $\mathrm{Z}_{0}^{*}(G)$ for the set of all such parameters.

One can show that $\mathrm{Z}_{0}^{*}(G) \neq \emptyset$ if and only if $G$ is quasi-split. In fact, we have the following more precise result, which is proved analogously to [3, Prop A.7].

Proposition 3.5.2. Let $\mathfrak{q} \subset \mathfrak{g}$ be a $\theta$-stable parabolic subalgebra. Choose a $\theta$-stable Levi decomposition

$$
\mathfrak{q}=\mathfrak{l} \oplus \mathfrak{u}
$$

The following are equivalent:

(i) $\mathfrak{g}_{0}$ is quasi-split and the $\mathbf{K}$-saturation of $\mathfrak{u} \cap \mathfrak{p}+\mathcal{N}_{\mathfrak{l}, \theta}$ has the same dimension as $\mathcal{N}_{\mathfrak{g}, \theta}$.

(ii) $\mathfrak{u} \cap \mathfrak{p}+\mathcal{N}_{\mathfrak{l}, \theta}$ contains a principal nilpotent element of $\mathfrak{g}$.

(iii) There is a maximally compact $\theta$-stable Cartan subalgebra $\mathfrak{h}^{\text {comp }} \subset \mathfrak{l}$ and a large, type $Z$ system $\Delta^{+}\left(\mathfrak{g}, \mathfrak{h}^{c}\right)$, compatible with $\mathfrak{q}$.

(iv) There is a maximally split $\theta$-stable Cartan subalgebra $\mathfrak{h}^{\text {split }} \subset \mathfrak{l}$ and a large, type $Z$ system $\Delta^{+}\left(\mathfrak{g}, \mathfrak{h}^{s}\right)$, compatible with $\mathfrak{q}$. 
Suppose $L$ is a quasi-split real reductive group and $B \subset L$ is real Borel subgroup. Let $S_{0}(L)$ be the Harish-Chandra module of the spherical principal series representation of $L$ of infinitesimal character 0

$$
S_{0}(L):=\text { Harish-Chandra module of } \operatorname{Ind}_{B}^{L} \mathbb{C} .
$$

By [14, Thm 1], $S_{0}(L)$ is irreducible and

$$
S_{0}(L) \simeq_{\mathbf{K}} \mathbb{C}\left[\mathcal{N}_{\mathfrak{l}, \theta}\right] .
$$

This implies (see e.g. [5, Cor 6.4]) that $\operatorname{AV}\left(S_{0}(L)\right)=\mathcal{N}_{\mathfrak{l}, \theta}$. Thus, $\left[S_{0}(L)\right] \in$ $\operatorname{Unip}_{\mathcal{O}}^{R}(L)$. If $\left(\mathfrak{l}, \mathfrak{q}, \chi^{\#}\right)$ is a triple as in Definition 3.5.1, define

$$
\widetilde{I}\left(\mathfrak{l}, \mathfrak{q}, \chi^{\#}\right):=I\left(\mathfrak{l}, \mathfrak{q}, \chi^{\#} \otimes S_{0}(L)\right) \in K_{0}(\mathfrak{g}, \mathbf{K}) .
$$

The assignment $\left(\mathfrak{l}, \mathfrak{q}, \chi^{\#}\right) \mapsto \widetilde{I}\left(\mathfrak{l}, \mathfrak{q}, \chi^{\#}\right)$ is constant on $\mathbf{K}$-conjugacy classes and thus induces a function

$$
\widetilde{I}: \mathrm{Z}_{0}(G) \rightarrow K_{0}(\mathfrak{g}, \mathbf{K})
$$

Next, we define a function $Z: \mathrm{BB}_{0}^{*}(G) \rightarrow \mathrm{Z}_{0}(G)$ which commutes with $I$ and $\widetilde{I}$. This requires a lemma.

Lemma 3.5.4 ([2], Lemma 16.1.4). Let $H \subset G$ be a $\theta$-stable Cartan subgroup. A character $\chi$ of $H$ is an extremal weight of an irreducible, finite-dimensional representation of $G$ if and only if

(i) $\left\langle d \chi, \alpha^{\vee}\right\rangle \in \mathbb{Z}$ for every root $\alpha \in \Delta(\mathfrak{g}, \mathfrak{h})$, and

(ii) $\chi\left(m_{\alpha}\right)=(-1)^{\left\langle d \chi, \alpha^{\vee}\right\rangle}$ for every real root $\alpha \in \Delta(\mathfrak{g}, \mathfrak{h})$

Suppose $[\mathfrak{h}, \mathfrak{b}, \chi] \in \operatorname{BB}_{0}^{*}(G)$. Form the parabolic subalgebra $\mathfrak{q}^{Z}=\mathfrak{l}^{Z} \oplus \mathfrak{u}^{Z} \subset \mathfrak{g}$ as in (3.1.5). By Definition 3.1.8 (iii), every simple real root $\alpha \in \Delta^{+}(\mathfrak{g}, \mathfrak{h})$ is even for $\chi$. Hence, every real root is even for $\chi$ (see [18, Cor 4.3.20]). Define a second character of $H$ by

$$
\chi^{Z}:=\chi \otimes\left|\rho\left(\mathfrak{n} \cap \mathfrak{l}^{Z}\right)\right| .
$$

Since $\left|\rho\left(\mathfrak{n} \cap \mathfrak{l}^{Z}\right)\right|$ takes positive values on $H$, every real root is even for $\chi^{Z}$. Furthermore

$$
d \chi^{Z}=-\rho(\mathfrak{n})+\rho\left(\mathfrak{n} \cap \mathfrak{l}^{Z}\right)=-\rho\left(\mathfrak{u}^{Z}\right) .
$$

Thus, $\left\langle d \chi^{Z}, \alpha^{\vee}\right\rangle=0$ for every root $\alpha \in \Delta\left(\mathfrak{l}^{Z}, \mathfrak{h}\right)$. Hence by Lemma 3.5.4, $\chi^{Z}$ is an extremal weight of an irreducible finite-dimensional representation of $L^{Z}$. By (3.5.5), this representation is a character. We will (somewhat abusively) denote this character (and its Harish-Chandra module) by $\chi^{Z}$.

It is easy to see that $\left[\mathfrak{l}^{Z}, \mathfrak{q}^{Z}, \chi^{Z}\right] \in \mathrm{Z}_{0}^{*}(G)$; condition (i) is immediate since all roots $\Delta\left(\mathfrak{l}^{Z}, \mathfrak{h}\right)$ are real. Condition (ii) follows from Definition 3.1.8(ii) and Proposition 3.1.7. Condition (iii) follows from (3.5.5). Condition (iv) is a consequence of Definition 3.1.8(i) and Proposition 3.5.2, Now define

$$
Z: \mathrm{BB}_{0}^{*}(G) \rightarrow \mathrm{Z}_{0}^{*}(G) \quad Z[\mathfrak{h}, \mathfrak{b}, \chi]=\left[\mathfrak{l}^{Z}, \mathfrak{q}^{Z}, \chi^{Z}\right] .
$$

Proposition 3.5.6. The function $Z: \mathrm{BB}_{0}^{*}(G) \rightarrow \mathrm{Z}_{0}^{*}(G)$ is surjective and the following diagram commutes

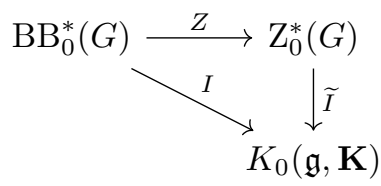


Proof. Suppose $\left[\mathfrak{l}, \mathfrak{q}, \chi^{\#}\right] \in \mathrm{Z}_{0}^{*}(G)$. By Proposition 3.5.2, there is a maximally split $\theta$-stable Cartan subalgebra $\mathfrak{h} \subset \mathfrak{l}$ and large, type Z system $\Delta^{+}(\mathfrak{g}, \mathfrak{h})$, compatible with $\mathfrak{q}$. Let $\mathfrak{b}$ be the corresponding Borel subalgebra of $\mathfrak{g}$. Define a character $\chi$ of $H$ by

$$
\chi:=\left.\chi^{\#}\right|_{H} \otimes|\rho(\mathfrak{n} \cap \mathfrak{l})|^{-1} .
$$

Then $[\mathfrak{h}, \mathfrak{b}, \chi] \in \mathrm{BB}_{0}^{*}(G)$ (conditions (i) and (ii) are automatic. Condition (iii) follows from Lemma 3.5.4). Furthermore $Z[\mathfrak{h}, \mathfrak{b}, \chi]=\left[\mathfrak{l}, \mathfrak{q}, \chi^{\#}\right]$. Hence, $Z$ is surjective onto $\mathrm{Z}_{0}^{*}(G)$. .

Now suppose $[\mathfrak{h}, \mathfrak{b}, \chi] \in \operatorname{BB}_{0}^{*}(G)$. Let $B^{L} \subset L^{Z}$ be the real Borel subgroup corresponding to $\mathfrak{l}^{Z} \cap \mathfrak{b}$. There is an identification

$$
I_{\left(\mathfrak{l}^{Z} \cap \mathfrak{b}, T\right)}^{\left(\mathfrak{l}^{Z}, L^{Z} \cap K\right)} \chi \simeq \text { Harish-Chandra module of } \operatorname{Ind}_{B^{L}}^{L^{Z}} \chi^{Z} .
$$

Since $\chi^{Z}$ extends to a character of $L^{Z}$

$$
\operatorname{Ind}_{B^{L}}^{L^{Z}} \chi^{Z} \simeq \chi^{Z} \otimes \operatorname{Ind}_{B^{L}}^{L^{Z}} \mathbb{C}=\chi^{Z} \otimes S_{0}\left(L^{Z}\right) .
$$

Now use the transitivity of induction to deduce

$$
\begin{aligned}
I[\mathfrak{h}, \mathfrak{b}, \chi] & =I\left[\mathfrak{l}^{Z}, \mathfrak{q}^{Z}, I\left[\mathfrak{h}, \mathfrak{b}^{\mathfrak{l}}, \chi\right]\right] \\
& =I\left[\mathfrak{l}^{Z}, \mathfrak{q}^{Z}, \chi^{Z} \otimes S_{0}\left(L^{Z}\right)\right] \\
& =\widetilde{I}\left[\mathfrak{l}^{Z}, \mathfrak{q}^{Z}, \chi^{Z}\right] .
\end{aligned}
$$

\subsection{Main results.}

Proposition 3.6.1. Let $[\mathfrak{h}, \mathfrak{b}, \chi] \in \mathrm{BB}_{0}(G)$. Then $I[\mathfrak{h}, \mathfrak{b}, \chi] \neq 0$ if and only if $[\mathfrak{h}, \mathfrak{b}, \chi]$ is large (cf. Definition [3.1.4).

Proof. We begin by making several simplifications. First, note that every parameter $[\mathfrak{h}, \mathfrak{b}, \chi] \in \mathrm{BB}_{0}(G)$ can be made to be type $\mathrm{L}$ through a sequence of cross actions through complex simple roots $\alpha$ satisfying $\theta(\alpha) \in-\Delta^{+}(\mathfrak{g}, \mathfrak{h})$ (this is proved by an easy induction on the number of such simple roots). By Theorem 3.4.4, $I[\mathfrak{h}, \mathfrak{b}, \chi]$ is preserved (up to signs) under this chosen sequence of cross actions. Thus, we can assume that $[\mathfrak{h}, \mathfrak{b}, \chi]$ is type $L$. Next, form the parabolic subalgebra $\mathfrak{q}^{L}=\mathfrak{l}^{L} \oplus \mathfrak{u}^{L} \subset \mathfrak{g}$ as in (3.1.6). By Proposition 3.1.7, $\mathfrak{q}^{L}$ is $\sigma$-stable. By the transitivity of induction $I[\mathfrak{h}, \mathfrak{b}, \chi]=I\left[\mathfrak{l}^{L}, \mathfrak{q}^{L}, I\left[\mathfrak{h}, \mathfrak{l}^{L} \cap \mathfrak{b}, \chi\right]\right]$. Note that

$$
\left\langle d \chi+\rho\left(\mathfrak{n} \cap \mathfrak{l}^{L}\right), \alpha^{\vee}\right\rangle=\left\langle-\rho\left(\mathfrak{u}^{L}\right), \alpha^{\vee}\right\rangle=0 \quad \forall \alpha \in \Delta^{+}\left(\mathfrak{l}^{L}, \mathfrak{h}\right) .
$$

Thus by (2.2.4), the inner module $I\left[\mathfrak{h}, \mathfrak{l}^{L} \cap \mathfrak{b}, \chi\right]$ is either irreducible or 0 . Since $\mathfrak{q}^{L}$ is $\sigma$-stable, $I\left[\mathfrak{l}^{L}, \mathfrak{q}^{L}, \cdot\right]$ takes nonzero values on irreducible classes. Thus, replacing $\mathfrak{g}$ with $\mathfrak{l}^{L}$ if necessary, we can assume $\Delta(\mathfrak{g}, \mathfrak{h})$ has only imaginary roots.

Now, assume $I[\mathfrak{h}, \mathfrak{b}, \chi]=0$. Choose a positive system for $\Delta(\mathfrak{k}, \mathfrak{t})$ compatible with $\mathfrak{b}$. If $-\rho(\mathfrak{n})+2 \rho(\mathfrak{n} \cap \mathfrak{p})$ is dominant for $\Delta^{+}(\mathfrak{k}, \mathfrak{t})$, then by examination of lowest $\mathbf{K}$-types (see [13, Thm 10.44]), $I[\mathfrak{h}, \mathfrak{b}, \chi] \neq 0$, a contradiction. Hence, there is a simple compact root $\alpha \in \Delta^{+}(\mathfrak{k}, \mathfrak{t})$ with

$$
\begin{aligned}
0 & >\left\langle-\rho(\mathfrak{n})+2 \rho(\mathfrak{n} \cap \mathfrak{p}), \alpha^{\vee}\right\rangle \\
& =\left\langle\rho(\mathfrak{n})-2 \rho(\mathfrak{n} \cap \mathfrak{k}), \alpha^{\vee}\right\rangle \\
& =\left\langle\rho(\mathfrak{n}), \alpha^{\vee}\right\rangle-2\left\langle\rho(\mathfrak{n} \cap \mathfrak{k}), \alpha^{\vee}\right\rangle \\
& =\left\langle\rho(\mathfrak{n}), \alpha^{\vee}\right\rangle-2 .
\end{aligned}
$$


Since $\left\langle\rho(\mathfrak{n}), \alpha^{\vee}\right\rangle$ is an integer, this forces $\left\langle\rho(\mathfrak{n}), \alpha^{\vee}\right\rangle \leq 1$, and hence $\left\langle\rho(\mathfrak{n}), \alpha^{\vee}\right\rangle=1$, which implies that $\alpha$ is simple for $\Delta^{+}(\mathfrak{g}, \mathfrak{h})$.

Conversely, if there is a compact simple root, then $I[\mathfrak{h}, \mathfrak{b}, \chi]=0$ by a character identity of Schmid ([16, Thm 1).

Proposition 3.6.2. Let $\left[\mathfrak{l}, \mathfrak{q}, \chi^{\#}\right] \in \mathrm{Z}_{0}^{*}(G)$. Then there is a nonzero irreducible $(\mathfrak{g}, \mathbf{K})$-module $X$ of infinitesimal character 0 and a sign $\epsilon[\mathfrak{l}, \mathfrak{q}, \chi] \in\{ \pm 1\}$ such that $\epsilon[\mathfrak{l}, \mathfrak{q}, \chi] \widetilde{I}\left[\mathfrak{l}, \mathfrak{q}, \chi^{\#}\right]=[X]$. Furthermore

(i) $\operatorname{AV}(X)=\mathbf{K}\left(\mathfrak{u} \cap \mathfrak{p}+\mathcal{N}_{\mathfrak{l}, \theta}\right)$.

(ii) $\operatorname{AV}(\operatorname{Ann}(X)))=\mathcal{N}$.

In particular, $\epsilon[\mathfrak{l}, \mathfrak{q}, \chi] \widetilde{I}\left[\mathfrak{l}, \mathfrak{q}, \chi^{\#}\right] \in \operatorname{Unip}_{\mathcal{O}}^{R}(G)$.

Proof. Note that $S_{0}(L)$ is an irreducible $(\mathfrak{l}, \mathbf{L} \cap \mathbf{K})$-module in the weakly good range (cf. (2.2.3) $)$. Hence by (2.2.4) there is an integer $t \in \mathbb{Z}_{\geq 0}$ such that

$$
R^{i} I_{(\mathfrak{l}, \mathbf{L} \cap \mathbf{K})}^{(\mathfrak{g}, \mathbf{K})} S_{0}(L) \otimes \chi^{\#}= \begin{cases}\text { irreducible or } 0 & i=t \\ 0 & i \neq t\end{cases}
$$

Let $X:=R^{t} I_{(\mathfrak{l}, \mathbf{L} \cap \mathbf{K})}^{(\mathfrak{g}, \mathbf{K})} S_{0}(L) \otimes \chi^{\#}$. By Proposition 3.5.6, there is a parameter $[\mathfrak{h}, \mathfrak{b}, \chi] \in \mathrm{BB}_{0}^{*}(G)$ such that $\widetilde{I}\left(\mathfrak{l}, \mathfrak{q}, \chi^{\#}\right)=I(\mathfrak{h}, \mathfrak{b}, \chi)$. Hence by Proposition 3.6.1. $(-1)^{t}[X]=\widetilde{I}\left(\mathfrak{l}, \mathfrak{q}, \chi^{\#}\right) \neq 0$.

Condition (i) follows from the well-known formula for the associated variety of a nonzero cohomologically induced module in the weakly good range, see (2.2.5). For any $X \in M(\mathfrak{g}, \mathbf{K}), \operatorname{AV}(\operatorname{Ann}(X))$ coincides with the $\mathbf{G}$-saturation of $\operatorname{AV}(X)$. Now (ii) follows from (i) and Definition 3.5.1(iv).

Let $\operatorname{Irrep}_{0}(G) \subset K(\mathfrak{g}, \mathbf{K})$ be the set of (isomorphism classes of) nonzero irreducible $(\mathfrak{g}, \mathbf{K})$-modules of infinitesimal character 0. By Proposition 3.6.2, there is a function

$$
\widetilde{I}_{\epsilon}: \mathrm{Z}_{0}^{*}(G) \rightarrow \operatorname{Irrep}_{0}(G) \quad \widetilde{I}_{\epsilon}\left[\mathfrak{l}, \mathfrak{q}, \chi^{\#}\right]:=\epsilon\left[\mathfrak{l}, \mathfrak{q}, \chi^{\#}\right] \widetilde{I}\left[\mathfrak{l}, \mathfrak{q}, \chi^{\#}\right]
$$

Similarly, define

$$
I_{\epsilon}: \operatorname{BB}_{0}^{*}(G) \rightarrow \operatorname{Irrep}_{0}(G) \quad I_{\epsilon}[\mathfrak{h}, \mathfrak{b}, \chi]:=\epsilon\left[\mathfrak{l}^{Z}, \mathfrak{q}^{Z}, \chi^{Z}\right] I[\mathfrak{h}, \mathfrak{b}, \chi]=\widetilde{I}_{\epsilon}\left[\mathfrak{l}^{Z}, \mathfrak{q}^{Z}, \chi^{Z}\right] .
$$

Proposition 3.6.3. The function $I_{\epsilon}: \mathrm{BB}_{0}^{*}(G) \rightarrow \operatorname{Irrep}_{0}(G)$ is a bijection.

Proof. Injectivity is an immediate consequence of Corollary 3.4.3. It remains to show that $I_{\epsilon}$ is surjective. Suppose $X \in \operatorname{Irrep}_{0}(G)$ and choose a minimal parabolic $Q^{\text {min }}=L^{\text {min }} U^{\text {min }} \subset G$. By the Casselman subrepresentation theorem, there is an irreducible finite-dimensional representation $V$ of $L^{\mathrm{min}}$ such that

$$
X \subseteq \mathbf{I}_{\left(\mathfrak{l}^{\min }, \mathbf{L}^{\min \cap \mathbf{K})}\right.}^{(\mathfrak{g}, \mathbf{K})} V .
$$

Since $X$ has infinitesimal character $0, V$ has infinitesimal character $-\rho(\mathfrak{u})$. Since $V$ is finite-dimensional, this means that $-\rho(\mathfrak{u})$ is nonsingular. Hence, $\mathfrak{l}^{\min }$ is a Cartan subalgebra $\left(\right.$ say $\left.\mathfrak{h}^{\prime}:=\mathfrak{l}^{\text {min }}\right), \mathfrak{q}^{\text {min }}$ is a Borel subalgebra (say $\mathfrak{b}^{\prime}:=\mathfrak{q}^{\text {min }}$ ), and $V$ is a character (say $\left.\chi^{\prime}:=V\right)$.

Consider the set of all parameters $S \subset \mathrm{BB}_{0}(G)$ be which can be obtained from $\left[\mathfrak{h}^{\prime}, \mathfrak{b}^{\prime}, \chi^{\prime}\right]$ through the following operations:

(1) Cayley transforms through odd simple real roots, and

(2) cross actions through complex simple roots $\alpha$ satisfying $\theta(\alpha) \in-\Delta^{+}(\mathfrak{g}, \mathfrak{h})$. 
Consider the set $D$ of all subsets $\Omega \subset S$ such that

(i) every parameter $[\mathfrak{h}, \mathfrak{b}, \chi] \in \Omega$ has $I[\mathfrak{h}, \mathfrak{b}, \chi] \neq 0$, and

(ii) there is a decomposition $I\left[\mathfrak{h}^{\prime}, \mathfrak{b}^{\prime}, \chi^{\prime}\right]=\sum_{[\mathfrak{h}, \mathfrak{b}, \chi] \in \Omega} \pm I[\mathfrak{h}, \mathfrak{b}, \chi]$ (for some collection of signs).

Note that $D$ is nonempty, since $\left\{\left[\mathfrak{h}^{\prime}, \mathfrak{b}^{\prime}, \chi^{\prime}\right]\right\} \in D$. Define the function

$$
\widetilde{d}: D \rightarrow \mathbb{Z}_{\geq 0} \quad \widetilde{d}(\Omega):=\sum_{[\mathfrak{h}, \mathfrak{b}, \chi] \in \Omega} d[\mathfrak{h}, \mathfrak{b}, \chi],
$$

where $d$ is defined as in (3.2.2). If $\Omega_{1}, \Omega_{2} \in D$, write $\Omega_{1} \leq \Omega_{2}$ if every parameter in $\Omega_{2}$ can be obtained from some parameter in $\Omega_{1}$ through operations (1) and (2). By Lemmas 3.2.3 and 3.3.1 $\widetilde{d}$ is strictly increasing with respect to $\leq$. Hence $\leq$ defines a partial order on $D$. Since $D$ is finite, there is a maximal element $\Omega^{*} \in D$.

By definition, there is a decomposition

$$
I\left[\mathfrak{h}^{\prime}, \mathfrak{b}^{\prime}, \chi^{\prime}\right]=\sum_{[\mathfrak{h}, \mathfrak{b}, \chi] \in \Omega^{*}} \pm I[\mathfrak{h}, \mathfrak{b}, \chi] .
$$

If $[\mathfrak{h}, \mathfrak{b}, \chi] \in \Omega^{*}$, then $[\mathfrak{h}, \mathfrak{b}, \chi]$ is large by Proposition 3.6.1 It is type Z and totally even by the maximality of $\Omega^{*}$. Thus, $[\mathfrak{h}, \mathfrak{b}, \chi] \in \mathrm{BB}_{0}^{*}(G)$ and hence $I_{\epsilon}[\mathfrak{h}, \mathfrak{b}, \chi] \in$ $\operatorname{Irrep}_{0}(G)$. Since $[X]$ is an irreducible summand in $I\left[\mathfrak{h}^{\prime}, \mathfrak{b}^{\prime}, \chi^{\prime}\right]$, this means that $[X]=I_{\epsilon}[\mathfrak{h}, \mathfrak{b}, \chi]$ for some $[\mathfrak{h}, \mathfrak{b}, \chi] \in \Omega^{*}$.

Our main result is now an immediate consequence of Propositions 3.5.6, 3.6.2, and 3.6 .3 .

Corollary 3.6.5. The inclusion $\operatorname{Unip}_{\mathcal{O}}^{R}(G) \subseteq \operatorname{Irrep}_{0}(G)$ is an equality, and there is a commutative triangle of bijections

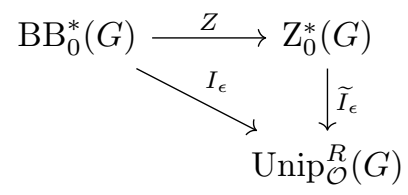

3.7. K-multiplicities of principal unipotent representations. Let $\left[\mathfrak{l}, \mathfrak{q}, \chi^{\#}\right] \in$ $\mathrm{Z}_{0}^{*}(G)$. Choose a maximal torus $\mathfrak{t} \subset \mathfrak{k}$ and a positive system $\Delta^{+}(\mathfrak{k}, \mathfrak{t})$ compatible with $\mathfrak{q}$. Consider the dot-action of $W_{\mathfrak{k}}$ on $\mathfrak{t}^{*}$ :

$$
w \cdot(\lambda)=w\left(\lambda+\rho_{\mathfrak{k}}\right)-\rho_{\mathfrak{k}} .
$$

If $\lambda \in \mathfrak{t}^{*}$, there is at most one element $w \in W_{\mathfrak{k}}$ such that $w \cdot \lambda$ is dominant for $\Delta^{+}(\mathfrak{k}, \mathfrak{t})$. Write $w_{\lambda}$ for this element (if it exists) and $\ell\left(w_{\lambda}\right)$ for its length. If $\lambda$ is integral (for $\Delta(\mathfrak{k}, \mathfrak{t})$ ) and dominant (for $\Delta^{+}(\mathfrak{k}, \mathfrak{t})$ ), write $V(\lambda)$ for the unique irreducible $\mathbf{K}$-representation with highest weight $\lambda$ (if $\lambda$ is not integral or dominant, put $V(\lambda)=0$ ). Consider the multiset of $\mathfrak{t}$-weights

$$
\Lambda\left(\mathfrak{l}, \mathfrak{q}, \chi^{\#}\right):=\Delta\left(2 \rho(\mathfrak{u} \cap \mathfrak{p})-\rho(\mathfrak{u}) \otimes \mathbb{C}\left[\mathcal{N}_{\mathfrak{l}, \theta}\right] \otimes S[\mathfrak{u} \cap \mathfrak{p}], \mathfrak{t}\right) .
$$

The following result is an easy consequence of the Blattner formula and Kostant's formula for the $\mathbf{K}$-structure of $S_{0}(L)$ (cf. 3.5.3).

Proposition 3.7.1. Let $\left[\mathfrak{l}, \mathfrak{q}, \chi^{\#}\right] \in \mathrm{Z}_{0}^{*}(G)$. Then

$$
I\left[\mathfrak{l}, \mathfrak{q}, \chi^{\#}\right] \simeq_{\mathbf{K}} \sum_{\lambda \in \Lambda\left(\mathfrak{l}, \mathfrak{q}, \chi^{\#}\right)}(-1)^{\ell\left(w_{0}\right)+\ell\left(w_{\lambda}\right)}\left[V\left(w_{\lambda} \cdot \lambda\right)\right] .
$$




\section{LANGLANDS PARAMETERS OF PRINCIPAL UNIPOTENT REPRESENTATIONS}

We begin by reviewing the Langlands classification of irreducible representations of real reductive groups, as formulated in 11. For details and proofs, we refer the reader to [1, Sec 4-5] or [4, Sec 2-3]. Let $\mathbf{G}$ be a complex connected reductive algebraic group and form the corresponding based root datum

$$
\Phi(\mathbf{G})=\left(X^{*}, X_{*}, \Delta, \Delta^{\vee}, \vee, \Pi, \Pi^{\vee}\right)
$$

(these symbols denote, respectively: the character lattice, the co-character lattice, the roots, the co-roots, the bijection between them, the simple roots, and the simple co-roots. This seven-tuple appears to depend on a choice of maximal torus and Borel. Up to canonical isomorphism, it does not). Let $\operatorname{Aut}(\mathbf{G})$ be the group of (algebraic group) automorphisms of $\mathbf{G}$ and $\operatorname{let} \operatorname{Int}(\mathbf{G}) \subset \operatorname{Aut}(\mathbf{G})$ be the normal subgroup of inner automorphisms. Two automorphisms $\theta, \theta^{\prime} \in \operatorname{Aut}(\mathbf{G})$ are inner if they are in the same (left) $\operatorname{Int}(\mathbf{G})$-coset. There is a canonical map $\operatorname{Aut}(\mathbf{G}) \rightarrow$ $\operatorname{Aut}(\Phi(\mathbf{G}))$, inducing a short exact sequence

$$
1 \rightarrow \operatorname{Int}(\mathbf{G}) \rightarrow \operatorname{Aut}(\mathbf{G}) \rightarrow \operatorname{Aut}(\Phi(\mathbf{G})) \rightarrow 1 .
$$

Hence, the inner classes in $\operatorname{Aut}(\mathbf{G})$ are parameterized by automorphisms of $\Phi(\mathbf{G})$.

Now fix a pinning $\left(\mathbf{h}, \mathfrak{b},\left\{X_{\alpha}\right\}\right)$ of $\mathbf{G}$ (by this we mean a Cartan $\mathfrak{h}$, a Borel $\mathfrak{b} \supset \mathfrak{h}$, and a set $\left\{X_{\alpha}\right\} \subset \mathfrak{b}$ of simple root vectors). For each $\delta \in \operatorname{Aut}(\Phi(\mathbf{G}))$, there is a unique automorphism $\theta_{0} \in \operatorname{Aut}(\mathbf{G})$ in the inner class of $\delta$ which preserves the chosen pinning

$$
d \theta_{0}(\mathfrak{h})=\mathfrak{h} \quad d \theta_{0}(\mathfrak{b})=\mathfrak{b} \quad d \theta_{0}\left\{X_{\alpha}\right\}=\left\{X_{\alpha}\right\}
$$

This automorphism is called the distinguished automorphism in the inner class of $\delta$, and the assignment $\delta \mapsto \theta_{0}$ defines a splitting of (4.0.1).

The dual group $\mathbf{G}^{\vee}$ comes equipped with a canonical isomorphism of based root data $\Phi\left(\mathbf{G}^{\vee}\right) \simeq \Phi(\mathbf{G})^{\vee}$ and hence a canonical isomorphism of groups (called tranpose)

$$
t: \operatorname{Aut}(\Phi(\mathbf{G})) \simeq \operatorname{Aut}\left(\Phi\left(\mathbf{G}^{\vee}\right)\right) .
$$

For what follows, we will fix both a pinning $\left(\mathfrak{h}^{\vee}, \mathfrak{b}^{\vee},\left\{X_{\alpha^{\vee}}\right\}\right)$ of $\mathbf{G}^{\vee}$ and an involution $\delta \in \operatorname{Aut}(\Phi(\mathbf{G}))$. Let $w_{0} \in W(\mathbf{G})$ be the longest element of the Weyl group. Note that $w_{0}^{2}=1$ and $w_{0}(\Pi)=-\Pi$. Hence $-w_{0}$ can be regarded as an involution of $\Phi\left(\mathbf{G}^{\vee}\right)$ (and as such, it commutes with every element of $\operatorname{Aut}\left(\Phi\left(\mathbf{G}^{\vee}\right)\right)$ ). We will consider the involution $\delta^{\vee}:=-w_{0} \delta^{t} \in \operatorname{Aut}\left(\Phi\left(\mathbf{G}^{\vee}\right)\right)$. As explained in the previous paragraph, there is a unique distinguished involution $\theta_{0}^{\vee} \in \operatorname{Aut}\left(\mathbf{G}^{\vee}\right)$ in the inner class of $\delta^{\vee}$.

The $L$-group of $\mathbf{G}$ (with respect to $\delta$ ) is the semi-direct product

$$
\mathbf{G}^{L}:=\mathbf{G}^{\vee} \ltimes\left\{1, \theta_{0}^{\vee}\right\} .
$$

The Weil group of $\mathbb{R}$ is the Lie group defined by

$$
W_{\mathbb{R}}:=\left\langle\mathbb{C}^{\times}, j\right\rangle \quad j z j^{-1}=\bar{z}, \quad j^{2}=-1 .
$$

Definition 4.0.2 ([15], see also [9], Sec 8.2). A Langlands parameter for $\mathbf{G}$ is a $\mathbf{G}^{\vee}$-conjugacy class of continuous homomorphisms $\phi: W_{\mathbb{R}} \rightarrow \mathbf{G}^{L}$ such that

(i) $\phi\left(\mathbb{C}^{\times}\right)$consists of semisimple elements.

(ii) $\phi(j) \in \mathbf{G}^{\vee} \theta_{0}^{\vee}$.

Denote the $\mathbf{G}^{\vee}$-conjugacy class of $\phi$ by $[\phi]$, and denote the set of Langlands parameters for $\mathbf{G}$ by $\Pi(\mathbf{G})$. 
Now let $G$ be a real form of $\mathbf{G}$. Assume that the Cartan involution $\theta \in \operatorname{Aut}(\mathbf{G})$ is in the inner class of $\delta$.

Theorem 4.0.3 (Langlands [15]). There is a natural map

$$
\varphi: \operatorname{Irrep}(G) \rightarrow \Pi(\mathbf{G}) .
$$

The fibers of this map are called L-packets in $\operatorname{Irrep}(G)$. If $G$ is quasi-split, then this map is surjective (i.e. all L-packets are non-empty).

The construction of $\varphi$ is quite technical - we will not repeat it here. A good overview can be found in [9, Sec 11]. We will use only the following observation, which is a trivial consequence of the construction.

Proposition 4.0.4. $\varphi\left(\operatorname{Irrep}_{0}(G)\right)=\left\{[\phi] \in \Pi(\mathbf{G}) \mid \phi\left(\mathbb{C}^{\times}\right)=\{1\}\right\}$.

Denote the set of parameters appearing in Proposition 4.0 .4 by $\Pi_{0}(\mathbf{G})$. If $X \in$ $\operatorname{Irrep}_{0}(G)$ is constructed from a Beilinson-Bernstein parameter $[\mathfrak{h}, \mathfrak{b}, \chi]$ (cf. Section [3.4), then there is an explicit formula in [3, Sec 3] for the Langlands parameter of $X$ in terms of $\mathfrak{h}, \mathfrak{b}$, and $\chi$. By Corollary 3.6.5, every $X \in \operatorname{Irrep}_{0}(G)$ is constructed from a uniquely defined $[\mathfrak{h}, \mathfrak{b}, \chi] \in \mathrm{BB}_{0}^{*}(G)$. If we apply the Adams-Vogan formula to the subset $\mathrm{BB}_{0}^{*}(G) \subset \mathrm{BB}_{0}(G)$, we obtain Proposition 4.0.5.

Proposition 4.0.5. An element $y \in N_{\mathbf{G}^{\vee} \theta_{0}^{\vee}}\left(\mathbf{H}^{\vee}\right)$ is called principal unipotent if

(i) $y^{2}=1$ (hence, $\operatorname{Ad}(y)$ defines an involution of $\mathbf{G}^{\vee}$ which preserves $\mathbf{H}^{\vee}$ ).

(ii) $\mathfrak{b}^{\vee}$ is small for $\operatorname{Ad}(y)$ (see Definition $3.1 .4($ ii)).

(iii) $\mathfrak{b}^{\vee}$ is type L for $\operatorname{Ad}(y)$ (see Definition 3.1.4(iii)).

There is a natural bijection

$\left\{\right.$ principal unipotent elements $\left.y \in N_{\mathbf{G}^{\vee} \theta_{0}^{\vee}}\left(\mathbf{H}^{\vee}\right)\right\} / \mathbf{H}^{\vee} \simeq \Pi_{0}(\mathbf{G})$.

Under this correpondence, $y \in N_{\mathbf{G}^{\vee} \theta_{0}^{\vee}}\left(\mathbf{H}^{\vee}\right)$ maps to the Langlands parameter $[\phi] \in$ $\Pi_{0}(\mathbf{G})$ defined by $\phi(j)=y$.

Specializing Proposition 4.0.5 to the case when $G$ is split (and hence $\theta_{0}^{\vee}=\mathrm{id}$ ), we obtain what appears to be a novel set of representatives for conjugacy classes of order-2 elements in a complex connected reductive algebraic group.

Corollary 4.0.6. There is a natural bijection

$$
\begin{aligned}
\left\{y \in N\left(\mathbf{H}^{\vee}\right) \mid y^{2}=1, \mathfrak{b}^{\vee} \text { is small and type } L \text { for } \operatorname{Ad}(y)\right\} / \mathbf{H}^{\vee} & \\
& \simeq\left\{y \in \mathbf{G}^{\vee} \mid y^{2}=1\right\} / \mathbf{G}^{\vee} .
\end{aligned}
$$

\section{REFERENCES}

[1] Jeffrey Adams, Dan Barbasch, and David A. Vogan Jr., The Langlands classification and irreducible characters for real reductive groups, Progress in Mathematics, vol. 104, Birkhäuser Boston, Inc., Boston, MA, 1992, DOI 10.1007/978-1-4612-0383-4. MR.1162533

[2] Jeffrey D. Adams, Marc A. A. van Leeuwen, Peter E. Trapa, and David A. Vogan Jr., Unitary representations of real reductive groups (English, with English and French summaries), Astérisque 417 (2020), viii + 188, DOI 10.24033/ast. MR 4146144

[3] Jeffrey Adams and David A. Vogan Jr., L-groups, projective representations, and the Langlands classification, Amer. J. Math. 114 (1992), no. 1, 45-138, DOI 10.2307/2374739. MR.1147719

[4] Jeffrey Adams and David A. Vogan Jr., Parameters for twisted representations, Representations of reductive groups, Progr. Math., vol. 312, Birkhäuser/Springer, Cham, 2015, pp. 51116. MR 3495793 
[5] J. Adams and D. Vogan. Associated varieties for real reductive groups, arXiv:2103.1183, 2021.

[6] James Arthur, On some problems suggested by the trace formula, Lie group representations, II (College Park, Md., 1982/1983), Lecture Notes in Math., vol. 1041, Springer, Berlin, 1984, pp. 1-49, DOI 10.1007/BFb0073144. MR748504

[7] James Arthur, Unipotent automorphic representations: conjectures, Astérisque 171-172 (1989), 13-71. Orbites unipotentes et représentations, II. MR 1021499

[8] Dan Barbasch and David A. Vogan Jr., Unipotent representations of complex semisimple groups, Ann. of Math. (2) 121 (1985), no. 1, 41-110, DOI 10.2307/1971193. MR782556

[9] A. Borel, Automorphic L-functions, Automorphic forms, representations and $L$-functions (Proc. Sympos. Pure Math., Oregon State Univ., Corvallis, Ore., 1977), Proc. Sympos. Pure Math., XXXIII, Amer. Math. Soc., Providence, R.I., 1979, pp. 27-61. MR.546608

[10] Henryk Hecht, Dragan Miličić, Wilfried Schmid, and Joseph A. Wolf, Localization and standard modules for real semisimple Lie groups. I. The duality theorem, Invent. Math. 90 (1987), no. 2, 297-332, DOI 10.1007/BF01388707. MR910203

[11] Ryoshi Hotta, Kiyoshi Takeuchi, and Toshiyuki Tanisaki, D-modules, perverse sheaves, and representation theory, Progress in Mathematics, vol. 236, Birkhäuser Boston, Inc., Boston, MA, 2008. Translated from the 1995 Japanese edition by Takeuchi, DOI 10.1007/978-0-81764523-6. MR2357361

[12] Anthony Joseph, On the associated variety of a primitive ideal, J. Algebra 93 (1985), no. 2, 509-523, DOI 10.1016/0021-8693(85)90172-3. MR786766

[13] Anthony W. Knapp and David A. Vogan Jr., Cohomological induction and unitary representations, Princeton Mathematical Series, vol. 45, Princeton University Press, Princeton, NJ, 1995, DOI 10.1515/9781400883936. MR1330919

[14] Bertram Kostant, On the existence and irreducibility of certain series of representations, Bull. Amer. Math. Soc. 75 (1969), 627-642, DOI 10.1090/S0002-9904-1969-12235-4. MR245725

[15] R. P. Langlands, On the classification of irreducible representations of real algebraic groups, Representation theory and harmonic analysis on semisimple Lie groups, Math. Surveys Monogr., vol. 31, Amer. Math. Soc., Providence, RI, 1989, pp. 101-170, DOI 10.1090/surv/031/03. MR1011897

[16] Wilfried Schmid, Two character identities for semisimple Lie groups, Non-commutative harmonic analysis (Actes Colloq., Marseille-Luminy, 1976), Springer, Berlin, 1977, pp. 196-225. Lecture Notes in Math., Vol. 587. MR0507247

[17] Peter E. Trapa, Annihilators and associated varieties of $A_{\mathfrak{q}}(\lambda)$ modules for $\mathrm{U}(p, q)$, Compositio Math. 129 (2001), no. 1, 1-45, DOI 10.1023/A:1013115223377. MR.1856021

[18] David A. Vogan Jr., Representations of real reductive Lie groups, Progress in Mathematics, vol. 15, Birkhäuser, Boston, Mass., 1981. MR632407

[19] David A. Vogan Jr., Associated varieties and unipotent representations, Harmonic analysis on reductive groups (Brunswick, ME, 1989), Progr. Math., vol. 101, Birkhäuser Boston, Boston, MA, 1991, pp. 315-388. MR.1168491

[20] David A. Vogan Jr., Irreducible characters of semisimple Lie groups. IV. Charactermultiplicity duality, Duke Math. J. 49 (1982), no. 4, 943-1073. MR683010

[21] Joseph A. Wolf, Finiteness of orbit structure for real flag manifolds, Geometriae Dedicata 3 (1974), 377-384, DOI 10.1007/BF00181328. MR364689

Mathematical Institute, University of Oxford, Andrew Wiles Building, Woodstock RoAd, OXford OX2 6GG ENGLAND

Email address: lucas.mason-brown@maths.ox.ac.uk 